\title{
Correction: Toosendanin demonstrates promising antitumor efficacy in osteosarcoma by targeting STAT3
}

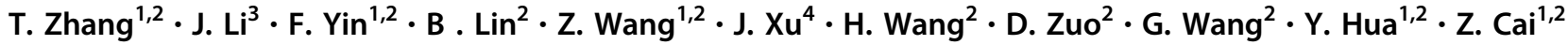

Published online: 15 February 2019

(c) Springer Nature Limited 2019

\section{Correction to: Oncogene;}

https://doi.org/10.1038/onc.2017.270;

Published online: 23 November 2017

Since the publication of this paper, the authors have noticed that the article contains an error in Fig. 3e (TSN,
$50 \mathrm{nM}$ ). As a result of the misfiling of the data, the incorrect image was inadvertently inserted in Fig. 3e during figure preparation. The corrected image is shown in this correction.
These authors contributed equally: T. Zhang, J. Li, F. Yin

The original article can be found online at https://doi.org/10.1038/onc. 2017.270.

T. Zhang

zhangtaoabc@2008.sina.com

$\triangle$ Y. Hua

hua_yingqi@163.com

$\triangle$ Z. Cai

czd856@vip.163.com

1 Department of Orthopedics, Shanghai General Hospital, Shanghai Jiao Tong University School of Medicine, Shanghai, China

2 Shanghai Bone Tumor Institution, Shanghai, China

3 Institute of Translational Medicine, Shanghai General Hospital, Shanghai Jiao Tong University School of Medicine, Shanghai, China

4 Department of Pharmacology, Institute of Medical Sciences, Shanghai Jiao Tong University School of Medicine,

Shanghai, China 
a
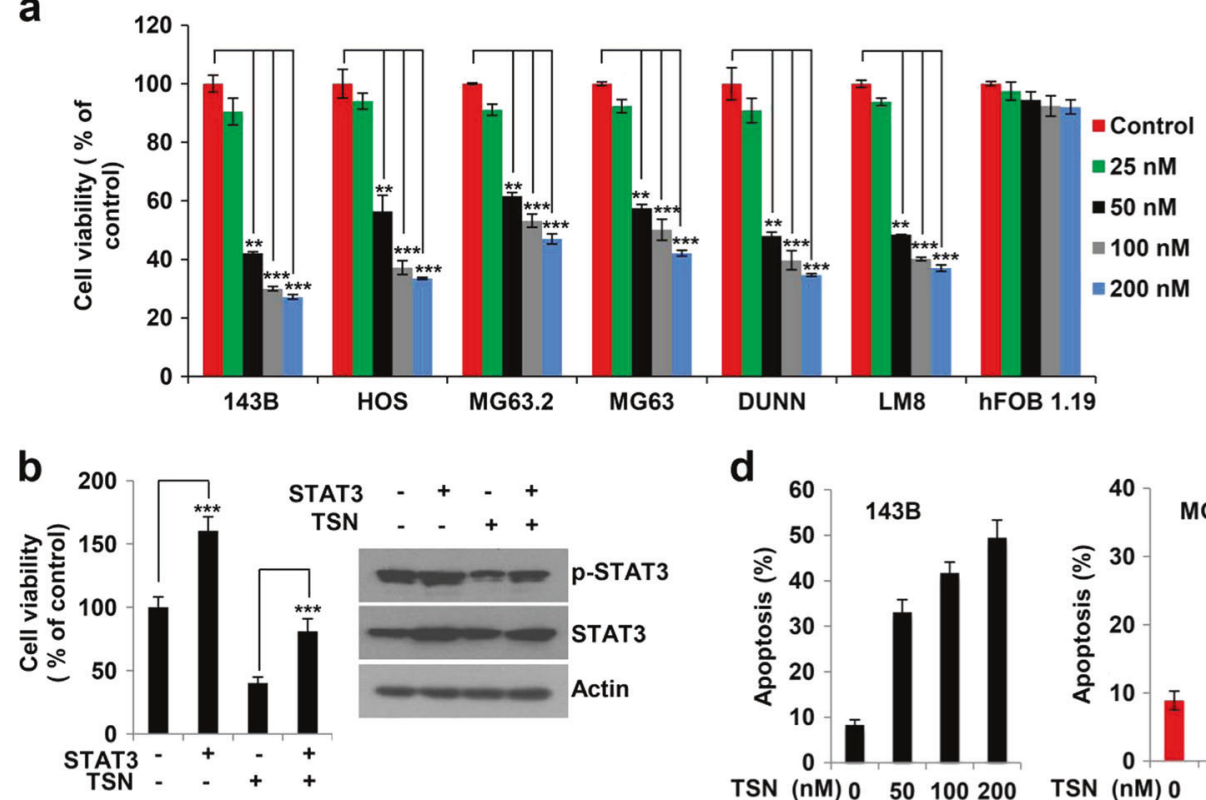

d
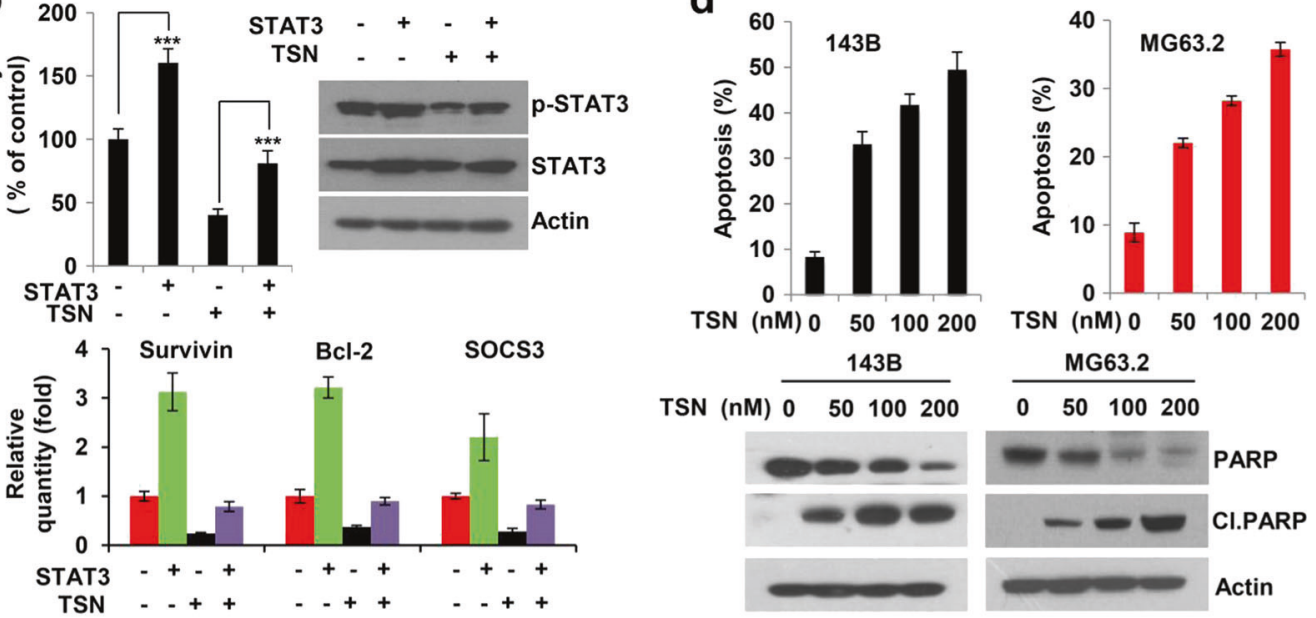

MG63.2

TSN (nM) $0 \quad 50100200$

$0 \quad 50 \quad 100200$
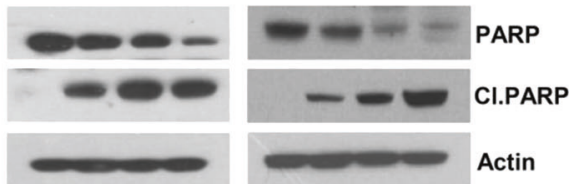

C

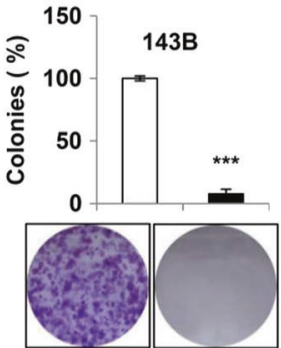

Control TSN (25 nM)
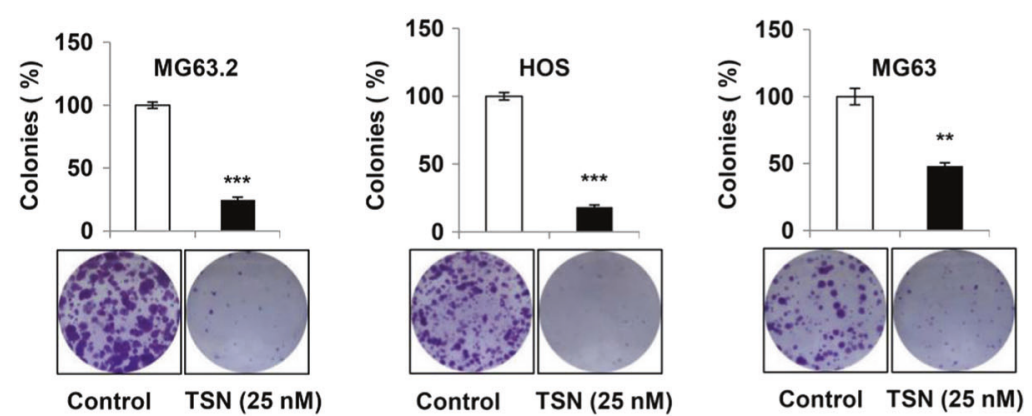

e

Condition Medium (CM)
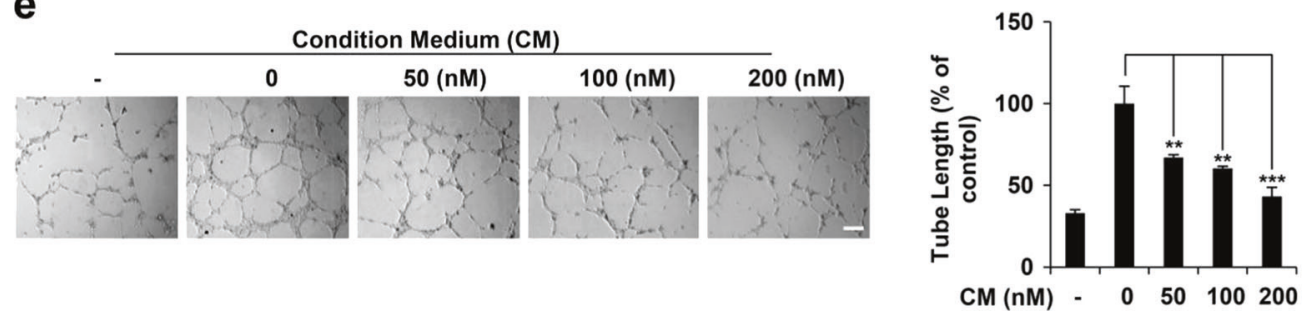

Fig. 3 TSN suppresses osteosarcoma cells growth and angiogenesis. (a) Osteosarcoma cell lines or normal osteoblast cell hFOB1.19 was exposed to indicated concentrations of TSN for $48 \mathrm{~h}$ and a MTS assay was performed. The bars indicate the mean \pm s.d. Statistically significant differences (Student's t-test), $* * P<0.01 ; * * * P<0.001$. (b) 143B cells were transfected with STAT3 vector, and then a MTS, western blot and qPCR assay were performed. (c) Osteosarcoma cells were seeded into six-well plates and treated with or without $25 \mathrm{nM}$ TSN for a week. Colonies were then fixed, stained and photographed. Colony numbers were counted manually. The bars indicate the mean \pm s.d. Statistically significant differences (Student's t-test), ${ }^{*} * P<0.01$; $* * * P<0.001$. (d) Cells were treated with indicted concentrations of TSN for $48 \mathrm{~h}$. Cells were stained with Annexin V and 7AAD, and analyzed by flow cytometry. The protein level of PARP and cleaved PARP were detected via western blot assay. (e) Tube formation assay. HUVECs $(2 \times 104)$ were seeded on Matrigel with conditional medium. After incubation for $8 \mathrm{~h}$, photomicrographs were acquired with an inverted microscope (OLYMPUS). Scale bar, $40 \mu \mathrm{m}$. The bars indicate the mean \pm s.d. Statistically significant differences (Student's t-test), $* * P<0.01 ; * * * P<0.001$. 